Hossein Sabzrou $\cdot$ Massoud Tousi $\cdot$ Siamak Yassemi

\title{
Simplicial join via tensor product
}

Received: 26 January 2008 / Revised: 4 February 2008

Published online: 23 February 2008

\begin{abstract}
Let $\mathbb{k}$ be a field, and $M$ and $N$ two finitely generated graded modules over standard graded $\mathbb{k}$-algebras $A$ and $B$, respectively. We will study generalized, sequentially, almost, and approximately Cohen-Macaulay as well as clean, and pretty clean properties of the $A \otimes_{\mathbb{k}} B$-module $M \otimes_{\mathbb{k}} N$ through the corresponding properties of $M$ and $N$. The behavior of these properties with respect to the simplicial join of two simplicial (multi)complexes will be revealed as corollaries.
\end{abstract}

\section{Introduction}

Let $\Delta$ be an abstract simplicial complex on the vertex set $[n]:=\{1, \ldots, n\}$, and $\mathbb{k}$ a field. The Stanley-Reisner ring $\mathbb{k}[\Delta]$ of $\Delta$ over $\mathbb{k}$ is by definition the quotient ring $R / I_{\Delta}$ where $R=\mathbb{k}\left[x_{1}, \ldots, x_{n}\right]$ is the polynomial ring over $\mathbb{k}$, and $I_{\Delta}$ is a squarefree monomial ideal generated by all monomials $x_{i_{1}} \ldots x_{i_{r}}$ such that $\left\{i_{1}, \ldots, i_{r}\right\} \notin \Delta$. When we talk about algebraic properties of $\Delta$ we refer to those of its Stanley-Reisner ring. Let $\Delta^{\prime}$ be a second simplicial complex whose vertex set differs from $\Delta$. The simplicial join $\Delta * \Delta^{\prime}$ is defined to be the simplicial complex whose simplicies are of the form $\sigma \cup \sigma^{\prime}$ where $\sigma \in \Delta$ and $\sigma^{\prime} \in \Delta^{\prime}$.

The algebraic and combinatorial properties of the simplicial join $\Delta * \Delta^{\prime}$ through the properties of $\Delta$ and $\Delta^{\prime}$ have been studied by a number of authors (cf. $[2,6,13]$, and [21]). For instance, in [6], Fröberg used the (graded) $\mathbb{k}$-algebra isomorphism $\mathbb{k}\left[\Delta * \Delta^{\prime}\right] \simeq \mathbb{k}[\Delta] \otimes_{\mathbb{k}} \mathbb{k}\left[\Delta^{\prime}\right]$, and proved that the tensor product of two graded $\mathbb{k}$-algebras is Cohen-Macaulay (resp. Gorenstein) if and only if both of them are Cohen-Macaulay (resp. Gorenstein).

Our approach is in the same spirit as [6], that is, via tensor product, but in a more general setting. We assume that $A$ and $B$ are two standard graded $\mathbb{k}$-algebras, i.e., finitely generated non-negatively graded $\mathbb{k}$-algebras generated over $\mathbb{k}$ by elements

H. Sabzrou $(\varangle) \cdot$ M. Tousi - S. Yassemi: School of Mathematics, Institute for Studies in Theoretical Physics and Mathematics (IPM), P.O. Box 19395-5746, Tehran, Iran e-mail: hossein@ipm.ir

M. Tousi: Department of Mathematics, Shahid Beheshti University, P.O. Box 19839, Tehran, Iran. e-mail: mtousi@ipm.ir

S. Yassemi: Department of Mathematics, University of Tehran, P.O. Box 13145-448, Tehran, Iran.e-mail: yassemi@ipm.ir

Mathematics Subject Classification (2000): 13C13, 13C14, 13F55 
of degree 1 , and $M$ and $N$ are two finitely generated graded modules over $A$ and $B$, respectively. We study various sorts of Cohen-Macaulayness (cf. Sect. 2), cleanness (cf. Definition 3.1), and pretty cleanness (cf. Definition 4.1) of the $A \otimes_{\mathbb{R}} B$-module $M \otimes_{\mathbb{k}} N$ through the corresponding properties of $M$ and $N$.

In [2], the authors presented a purely combinatorial argument for showing that sequential Cohen-Macaulayness is preserved under simplicial join. In Sect. 2, we will give a more general algebraic version of this result which shows that the $A \otimes_{\mathbb{k}} B$-module $M \otimes_{\mathbb{k}} N$ is sequentially Cohen-Macaulay (cf. Theorem 2.11) if and only if both $M$ and $N$ are sequentially Cohen-Macaulay. We also consider other variations of Cohen-Macaulayness (such as Buchsbaumness) which are not well-behaved with respect to the simplicial join, in the sense that, in general they are not preserved by the simplicial join.

In [13, Corollary 2.9], the authors combinatorially proved that the join of two shellable simplicial complexes is shellable. On the other hand, we know by a result of Dress [3] that cleanness is an algebraic counterpart of shellability. Therefore, the tensor product of two clean Stanley-Reisner rings is clean if both of them are clean. In Sect. 3, we will show that if the $A$-module $M$ and the $B$-module $N$ are clean, then under a suitable hypothesis, the $A \otimes_{\mathbb{k}} B$-module $M \otimes_{\mathbb{k}} N$ is clean (cf. Theorem 3.3). As a corollary, we will show that for two arbitrary monomial ideals $I$ and $J$ in the polynomial rings $R=\mathbb{k}\left[x_{1}, \ldots, x_{n}\right]$ and $S=\mathbb{k}\left[y_{1}, \ldots, y_{m}\right]$, respectively, we have $R / I \otimes_{\mathbb{R}} S / J$ is clean if and only if both $R / I$ and $S / J$ are clean (cf. Corollary 3.8).

The simplicial complex $\Delta$ can be regarded as a subset of $\{0,1\}^{n}$ which is closed under going down, i.e., if $\mathbf{a} \in \Delta, \mathbf{b} \in\{0,1\}^{n}$, and $\mathbf{b} \leq \mathbf{a}$ (componentwise), then $\mathbf{b} \in \Delta$. Replacing $\{0,1\}^{n}$ by $\mathbb{N}^{n}$ in this definition where $\mathbb{N}$ is the set of natural numbers including 0 , we get the definition of a simplicial multicomplex which is due to Stanley [16]. To remedy a defect, Herzog and Popescu [8] improved the Stanley's definition slightly and generalized the classical concept of shellability to the simplicial multicomplexes. They also introduced the algebraic counterpart of this new notion of shellability which is called pretty cleanness. For two simplicial multicomplexes $\Gamma$ and $\Gamma^{\prime}$, we can define the simplicial join $\Gamma * \Gamma^{\prime}$ in a natural way (cf. Sect. 4). Our final goal in this paper is to show that the simplicial join of two shellable simplicial multicomplexes is shellable precisely when both of them are shellable. We will show that for two arbitrary monomial ideals $I$ and $J$ in the polynomial rings $R=\mathbb{k}\left[x_{1}, \ldots, x_{n}\right]$ and $S=\mathbb{k}\left[y_{1}, \ldots, y_{m}\right]$, respectively, we have $R / I \otimes_{\mathbb{k}} S / J$ is pretty clean if and only if both $R / I$ and $S / J$ are pretty clean (cf. Corollary 4.8).

\section{Some sorts of Cohen-Macaulayness}

Throughout this paper $\mathbb{k}$ will denote a field. Let $M$ and $N$ be two finitely generated graded modules over standard graded $\mathbb{k}$-algebras $A$ and $B$, respectively. In this section, we provide some necessary and sufficient conditions for generalized, sequentially, almost, and approximately Cohen-Macaulay properties of the $A \otimes_{\mathbb{k}} B$-module $M \otimes_{\mathbb{k}} N$ in terms of the corresponding properties of the modules $M$ and $N$. 
Our method in this section is heavily based on the following lemma which is an immediate consequence of the Künneth tensor formula (cf. [19, Theorem 9.3.2]).

Lemma 2.1. Let $A$ and $B$ be two $\mathbb{k}$-algebras. Assume that $M$ and $M^{\prime}$ are $A$-modules, and $N$ and $N^{\prime}$ are $B$-modules. Then for all $i \geq 0$, we have the isomorphism of $A \otimes_{\mathbb{k}} B$-modules

$$
\operatorname{Ext}_{A \otimes_{\mathbb{k}} B}^{i}\left(M \otimes_{\mathbb{k}} N, M^{\prime} \otimes_{\mathbb{k}} N^{\prime}\right) \simeq \bigoplus_{p+q=i} \operatorname{Ext}_{A}^{p}\left(M, M^{\prime}\right) \otimes_{\mathbb{k}} \operatorname{Ext}_{B}^{q}\left(N, N^{\prime}\right) .
$$

Proof. Let $\mathbb{F}$ be a free resolution of the $A$-module $M$, and $\mathbb{G}$ be a free resolution of the $B$-module $N$, respectively. Then $\mathbb{F} \otimes_{\mathbb{k}} \mathbb{G}$ is a free resolution of the $A \otimes_{\mathbb{k}}$ $B$-module $M \otimes_{\mathbb{k}} N$, by [19, Theorem 9.3.2]. We have the natural isomorphism of complexes

$$
\operatorname{Hom}_{A \otimes_{\mathbb{k}} B}\left(\mathbb{F} \otimes_{\mathbb{k}} \mathbb{G}, M^{\prime} \otimes_{\mathbb{k}} N^{\prime}\right) \simeq \operatorname{Hom}_{A}\left(\mathbb{F}, M^{\prime}\right) \otimes_{\mathbb{k}} \operatorname{Hom}_{B}\left(\mathbb{G}, N^{\prime}\right) .
$$

Hence, using the Künneth tensor formula (cf. [19, Theorem 9.3.2]) we have

$$
H^{i}\left(\operatorname{Hom}_{A \otimes_{\mathbb{k}} B}\left(\mathbb{F} \otimes_{\mathbb{k}} \mathbb{G}, M^{\prime} \otimes_{\mathbb{k}} N^{\prime}\right)\right) \simeq \bigoplus_{p+q=i} H^{p}\left(\operatorname{Hom}_{A}\left(\mathbb{F}, M^{\prime}\right)\right) \otimes H^{q}\left(\operatorname{Hom}_{B}\left(\mathbb{G}, N^{\prime}\right)\right),
$$

as required.

To emphasize the graded maximal ideal $\mathfrak{m}$ of a standard graded algebra $A$, we use the notation $(A, \mathfrak{m})$. As an application of Lemma 2.1, we have the following key observation.

Theorem 2.2. Let $M$ and $N$ be two finitely generated graded modules over standard graded $\mathbb{k}$-algebras $(A, \mathfrak{m})$ and $(B, \mathfrak{n})$, respectively. Assume that $\mathfrak{M}:=\mathfrak{m} \otimes_{\mathbb{k}}$ $B+A \otimes_{\mathbb{k}} \mathfrak{n}$. Then for all $i \geq 0$, we have the isomorphism of $A \otimes_{\mathbb{k}} B$-modules

$$
H_{\mathfrak{M}}^{i}\left(M \otimes_{\mathbb{k}} N\right) \simeq \bigoplus_{p+q=i} H_{\mathfrak{m}}^{p}(M) \otimes_{\mathbb{k}} H_{\mathfrak{n}}^{q}(N) .
$$

Proof. We assume that $A=R / I$ and $B=S / J$ where $R=\mathbb{k}\left[x_{1}, \ldots, x_{n}\right]$ and $S=$ $\mathbb{k}\left[y_{1}, \ldots, y_{m}\right]$ are polynomial rings over the field $\mathbb{k}$, and $I$ and $J$ are homogeneous ideals in $R$ and $S$, respectively. Then $T:=R \otimes_{\mathbb{k}} S=\mathbb{k}\left[x_{1}, \ldots, x_{n}, y_{1}, \ldots, y_{m}\right]$, and so $A \otimes_{\mathbb{R}} B \simeq T /(I T+J T)$. We have

$$
\begin{aligned}
\operatorname{Ext}_{T}^{i}\left(M \otimes_{\mathbb{k}} N, T\right) & \simeq \operatorname{Ext}_{R \otimes_{\mathbb{k}} S}^{i}\left(M \otimes_{\mathbb{k}} N, R \otimes_{\mathbb{k}} S\right) \\
& \simeq \bigoplus_{p+q=i} \operatorname{Ext}_{R}^{p}(M, R) \otimes_{\mathbb{k}} \operatorname{Ext}_{S}^{q}(N, S),
\end{aligned}
$$

where the second isomorphism follows from Lemma 2.1. Since the Matlis duality functor $(-)^{\vee}:=\operatorname{Hom}_{\mathbb{k}}(-, \mathbb{k})$ is well-behaved with respect to the finite direct sum $\oplus_{p+q=i}$ and the tensor product $\bigotimes_{\mathbb{R}}$, by the local duality theorem we get the isomorphism of $R \otimes_{\mathbb{k}} S$-modules

$$
H_{\left\langle x_{1}, \ldots, x_{n}, y_{1}, \ldots, y_{m}\right\rangle}^{i}\left(M \otimes_{\mathbb{R}} N\right) \simeq \bigoplus_{p+q=i} H_{\left\langle x_{1}, \ldots, x_{n}\right\rangle}^{p}(M) \otimes_{\mathbb{k}} H_{\left\langle y_{1}, \ldots, y_{m}\right\rangle}^{q}(N) .
$$

Now, the result follows from the independence theorem for local cohomology. 
Corollary 2.3. Let $M$ and $N$ be two finitely generated graded modules over standard graded $\mathbb{k}$-algebras $(A, \mathfrak{m})$ and $(B, \mathfrak{n})$, respectively. Then

(1) $\operatorname{dim}_{A \otimes_{\mathbb{k}} B}\left(M \otimes_{\mathbb{k}} N\right)=\operatorname{dim}_{A}(M)+\operatorname{dim}_{B}(N)$.

(2) $\operatorname{depth}_{A \otimes_{\mathbb{k}} B}\left(M \otimes_{\mathbb{k}} N\right)=\operatorname{depth}_{A}(M)+\operatorname{depth}_{B}(N)$.

Proof. Both statements follow from Theorem 2.2, and the equalities

$$
\operatorname{dim}_{A}(M)=\max \left\{i: H_{\mathfrak{m}}^{i}(M) \neq 0\right\},
$$

and

$$
\operatorname{depth}_{A}(M)=\min \left\{i: H_{\mathfrak{m}}^{i}(M) \neq 0\right\}
$$

respectively.

Lemma 2.4. Let $M$ and $N$ be two graded modules over standard graded $\mathbb{k}$-algebras $A$ and $B$, respectively. Assume that $M \otimes_{\mathbb{R}} N \neq 0$. Then the length $\ell_{A \otimes_{\mathbb{k}} B}\left(M \otimes_{\mathbb{R}} N\right)$ is finite if and only if both $\ell_{A}(M)$ and $\ell_{B}(N)$ are finite.

Proof. If both $\ell_{A}(M)$ and $\ell_{B}(N)$ are finite. Then both $M$ and $N$ are Noetherian and Artinian. Therefore $M \otimes_{\mathbb{k}} N$ is Noetherian, and $\operatorname{dim}_{A}(M)=\operatorname{dim}_{B}(N)=0$. It follows from Corollary 2.3 that $\operatorname{dim}_{A \otimes_{\mathbb{k}} B}\left(M \otimes_{\mathbb{k}} N\right)=0$ and so $M \otimes_{\mathbb{k}} N$ is Artinian, as required.

Conversely, suppose the contrary that $M$ is not Noetherian. Then there exists a strictly ascending chain of submodules of $M$ which is not stationary. Extending this chain of submodules in $M \otimes_{\mathbb{k}} N$, we conclude that $M \otimes_{\mathbb{k}} N$ is not Noetherian which is a contradiction. Moreover, since $\operatorname{dim}_{A \otimes_{\mathbb{k}} B}\left(M \otimes_{\mathbb{k}} N\right)=0$, we conclude that $\operatorname{dim}_{A}(M)=\operatorname{dim}_{B}(N)=0$, as desired.

Definition 2.5. Let $M$ be a finitely generated graded module over a standard graded $\mathbb{k}$-algebra $(A, \mathfrak{m})$ with $\operatorname{dim}_{A} M=d$. The $A$-module $M$ is called generalized CohenMacaulay if the length of the $A$-module $H_{\mathfrak{m}}^{i}(M)$ is finite for $i=0,1, \ldots, d-1$.

Theorem 2.6. Let $M$ and $N$ be two finitely generated graded modules over standard graded $\mathbb{k}$-algebras $(A, \mathfrak{m})$ and $(B, \mathfrak{n})$, respectively. Assume that both $\operatorname{dim}_{A} M$ and $\operatorname{dim}_{B} N$ are positive. Then the following conditions are equivalent:

(1) $M \otimes_{\mathbb{k}} N$ is generalized Cohen-Macaulay.

(2) $M \otimes_{\mathbb{k}} N$ is Buchsbaum.

(3) $M \otimes_{\mathbb{k}} N$ is Cohen-Macaulay.

(4) Both $M$ and $N$ are Cohen-Macaulay.

Proof. (4) $\Rightarrow$ (3): Follows from Corollary 2.3.

(3) $\Rightarrow(2)$ : Trivial.

(2) $\Rightarrow$ (1): Follows from [17, Corollary I.2.4].

$(1) \Rightarrow(4)$ : We assume that $\operatorname{dim}_{A} M=d$ and $\operatorname{dim}_{B} N=d^{\prime}$. Then by Corollary 2.3, we have $\operatorname{dim}_{A \otimes_{\mathbb{k}} B}\left(M \otimes_{\mathbb{k}} N\right)=d+d^{\prime}$. For each $i=0,1, \ldots, d+d^{\prime}-1$, we have $\ell\left(H_{\mathfrak{M}}^{i}\left(M \otimes_{\mathbb{k}} N\right)\right)<\infty$ where $\mathfrak{M}=\mathfrak{m} \otimes_{\mathbb{k}} B+A \otimes_{\mathbb{k}} \mathfrak{n}$. Let $0 \leq i \leq d^{\prime}-1$. Then by Theorem 2.2, $H_{\mathfrak{m}}^{d}(M) \otimes_{\mathbb{k}} H_{\mathfrak{n}}^{i}(N)$ is a direct summand of $H_{\mathfrak{M}}^{d+i}(M \otimes N)$ and 
so must be of finite length. Since $H_{\mathfrak{m}}^{d}(M)$ is not finitely generated, by Lemma 2.4, we conclude that $H_{\mathfrak{n}}^{i}(N)=0$ for all $0 \leq i \leq d^{\prime}-1$. This implies that $N$ is a Cohen-Macaulay $B$-module. Similarly, we can show that $M$ is a Cohen-Macaulay $A$-module.

Remark 2.7. If $\operatorname{dim}_{B} N=0$, then $\operatorname{dim}_{A \otimes_{\mathbb{k}} B} M \otimes_{\mathbb{k}} N=\operatorname{dim}_{A} M$, and for each $i \geq 0$, we have $H_{\mathfrak{M}}^{i}\left(M \otimes_{\mathbb{k}} N\right) \simeq H_{\mathfrak{m}}^{i}(M) \otimes_{\mathbb{k}} H_{\mathfrak{n}}^{0}(N)$. Consequently, $M \otimes_{\mathbb{k}} N$ is generalized Cohen-Macaulay if and only if $M$ is generalized Cohen-Macaulay.

Remark 2.8. The notion of generalized Cohen-Macaulay was introduced in [15]. For a simplicial complex $\Delta$ this notion coincides with the so-called notion of Buchsbaum. Buchsbaum simplicial complexes have several algebraic and combinatorial characterizations (cf. [12,17]). For instance, a simplicial complex $\Delta$ is Buchsbaum over a field $\mathbb{k}$ if and only if it is pure and locally Cohen-Macaulay (i.e., the link of each vertex is Cohen-Macaulay). Recall that the link of a face $\sigma \in \Delta$ is defined as $\operatorname{link}_{\Delta}(\sigma):=\{\tau \in \Delta \mid \sigma \cap \tau=\emptyset, \quad \sigma \cup \tau \in \Delta\}$. Using this characterization, we can see that the simplicial join of the triangulation of a cylinder (which is Buchsbaum [17, Example II.2.13(i)]) with a simplicial complex with only one vertex (which is Cohen-Macaulay [17, Example II.2.14(ii)] and so Buchsbaum) is not Buchsbaum.

Corollary 2.9. Let $\Delta$ and $\Delta^{\prime}$ be two simplicial complexes over disjoint vertex sets. Then $\Delta * \Delta^{\prime}$ is Buchsbaum (over $\mathbb{k}$ ) if and only if $\Delta$ and $\Delta^{\prime}$ are Cohen-Macaulay (over $\mathbb{k})$.

Definition 2.10 (Stanley [16]). Let $A$ be a standard graded $\mathbb{k}$-algebra. Let $M$ be a finitely generated graded $A$-module. We say that $M$ is sequentially Cohen-Macaulay if there exists a finite filtration (called Cohen-Macaulay filtration)

$$
0=M_{0} \subset M_{1} \subset \cdots \subset M_{r}=M
$$

of $M$ by graded submodules $M_{i}$ satisfying the two conditions:

(1) Each quotient $M_{i} / M_{i-1}$ is Cohen-Macaulay.

(2) $\operatorname{dim}\left(M_{1} / M_{0}\right)<\operatorname{dim}\left(M_{2} / M_{1}\right)<\cdots<\operatorname{dim}\left(M_{r} / M_{r-1}\right)$ where dim denotes Krull dimension.

Theorem 2.11. Let $A$ and $B$ be two standard graded $\mathbb{k}$-algebras. Let $M$ and $N$ be two finitely generated graded modules over $A$ and $B$, respectively. Then $M \otimes_{\mathbb{k}} N$ is sequentially Cohen-Macaulay $A \otimes_{\mathbb{k}} B$-module if and only if $M$ and $N$ are sequentially Cohen-Macaulay over $A$ and $B$, respectively.

Proof. We assume that $A=R / I$ and $B=S / J$ where $R=\mathbb{k}\left[x_{1}, \ldots, x_{n}\right]$ and $S=\mathbb{k}\left[y_{1}, \ldots, y_{m}\right]$ are polynomial rings over the field $\mathbb{k}$, and $I$ and $J$ are homogeneous ideals in $R$ and $S$, respectively. Let $T:=R \otimes_{\mathbb{k}} S=\mathbb{k}\left[x_{1}, \ldots, x_{n}, y_{1}, \ldots, y_{m}\right]$. Then $A \otimes_{\mathbb{k}} B \simeq T /(I T+J T)$. To prove the result, it is enough to show that $M \otimes_{\mathbb{k}} N$ is sequentially Cohen-Macaulay over $T$ if and only if $M$ and $N$ are sequentially Cohen-Macaulay over $R$ and $S$, respectively. 
First we assume that $M$ and $N$ are sequentially Cohen-Macaulay over $R$ and $S$, respectively. We show that $\operatorname{Ext}_{T}^{i}\left(M \otimes_{\mathbb{k}} N, T\right)$ is either 0 or Cohen-Macaulay of dimension $n+m-i$ which by [8, Theorem 2.4] will give us the result. We have

$$
\begin{aligned}
\operatorname{Ext}_{T}^{i}\left(M \otimes_{\mathbb{k}} N, T\right) & \simeq \operatorname{Ext}_{R \otimes_{\mathbb{k}} S}^{i}\left(M \otimes_{\mathbb{k}} N, R \otimes_{\mathbb{k}} S\right) \\
& \simeq \bigoplus_{p+q=i} \operatorname{Ext}_{R}^{p}(M, R) \otimes_{\mathbb{k}} \operatorname{Ext}_{S}^{q}(N, S),
\end{aligned}
$$

where the second isomorphism follows from Lemma 2.1. The $R$-module $\operatorname{Ext}_{R}^{p}(M, R)$ is either 0 or Cohen-Macaulay of dimension $n-p$ because $M$ is sequentially Cohen-Macaulay. Similarly, $\operatorname{Ext}_{S}^{q}(N, S)$ is either 0 or Cohen-Macaulay of dimension $m-q$. If both of them are non-zero, then by Corollary 2.3 and Theorem 2.6, $\operatorname{Ext}_{R}^{p}(M, R) \otimes_{\mathbb{k}} \operatorname{Ext}_{S}^{q}(N, S)$ is Cohen-Macaulay of dimension $(n-p)+$ $(m-q)=n+m-i$, as required.

Conversely, assume that $M \otimes_{\mathbb{k}} N$ is sequentially Cohen-Macaulay over $T=$ $R \otimes_{\mathbb{R}} S$. Assume in addition that $\operatorname{Ext}_{R}^{p}(M, R) \neq 0$. We prove that $\operatorname{Ext}_{R}^{p}(M, R)$ is Cohen-Macaulay of dimension $n-p$. There exist some integer $q$ with $\operatorname{codim}(N) \leq$ $q \leq \operatorname{proj}_{-\operatorname{dim}_{S}}(N)$ such that $\operatorname{Ext}_{S}^{q}(N, S) \neq 0$. Hence $\operatorname{Ext}_{R}^{p}(M, R) \otimes_{\mathbb{k}} \operatorname{Ext}_{S}^{q}(N, S)$ is a non-zero direct summand of the $(n+m)-(p+q)$-dimensional Cohen-Macaulay module $\operatorname{Ext}_{T}^{p+q}\left(M \otimes_{\mathbb{k}} N, T\right)$ and so we have

$$
\begin{aligned}
\operatorname{depth}\left(\operatorname{Ext}_{R}^{p}(M, R) \otimes_{\mathbb{k}} \operatorname{Ext}_{S}^{q}(N, S)\right) & \geq \operatorname{depth}\left(\operatorname{Ext}_{T}^{p+q}\left(M \otimes_{\mathbb{k}} N, T\right)\right) \\
& =\operatorname{dim}\left(\operatorname{Ext}_{T}^{p+q}\left(M \otimes_{\mathbb{k}} N, T\right)\right) \\
& \geq \operatorname{dim}\left(\operatorname{Ext}_{R}^{p}(M, R) \otimes_{\mathbb{k}} \operatorname{Ext}_{S}^{q}(N, S)\right) .
\end{aligned}
$$

Therefore $\operatorname{Ext}_{R}^{p}(M, R) \otimes_{\mathbb{k}} \operatorname{Ext}_{S}^{q}(N, S)$ is Cohen-Macaulay, and we have

$$
\operatorname{dim} \operatorname{Ext}_{R}^{p}(M, R)+\operatorname{dim}_{E^{2} t_{S}^{q}(N, S)=n+m-(p+q) .}
$$

Since $\operatorname{dim} \operatorname{Ext}_{R}^{p}(M, R) \leq n-p$ and $\operatorname{dim} \operatorname{Ext}_{S}^{q}(N, S) \leq m-q$, by [1, Corollary 3.5.11], we conclude that $\operatorname{dim} \operatorname{Ext}_{R}^{p}(M, R)=n-p$, as required.

Remark 2.12. We refer the reader to [4, Theorem 3.3], [16, Proposition II.2.10], and [20, Proposition 1.4] for three different combinatorial characterizations of the sequentially Cohen-Macaulay simplicial complexes.

Corollary 2.13. Let $\Delta$ and $\Delta^{\prime}$ be two simplicial complexes over disjoint vertex sets. Then $\Delta * \Delta^{\prime}$ is sequentially Cohen-Macaulay (over $\mathbb{k}$ ) if and only if $\Delta$ and $\Delta^{\prime}$ are sequentially Cohen-Macaulay (over $\mathbb{k}$ ).

Definition 2.14. Let $M$ be a finitely generated graded module over a standard graded $\mathbb{k}$-algebra $A$ with $\operatorname{dim}_{A}(M)=d$.

(1) The $A$-module $M$ is called almost Cohen-Macaulay if depth $M \geq d-1$.

(2) Let $\left\{N_{1}, \ldots, N_{n}\right\}$ denote a reduced primary decomposition of the $A$-module $M$ where each $N_{j}$ is a $\mathfrak{p}_{j}$-primary submodule of $M$. Let

$$
\mathcal{U}_{M}(0):=\bigcap_{\operatorname{dim} A / \mathfrak{p}_{j}=d} N_{j} .
$$


The $A$-module $M$ is called approximately Cohen-Macaulay whenever it is almost Cohen-Macaulay and $M / \mathcal{U}_{M}(0)$ is Cohen-Macaulay.

Remark 2.15. The notion of almost Cohen-Macaulay has had different meanings in the literature. The definition here, was obtained from [10]. The original definition of the approximately Cohen-Macaulay property was given by Goto [7] for rings. The definition here, was taken from [14, Definition 4.4].

Theorem 2.16 ([14, Proposition 4.5]). Let $M$ be a finitely generated graded module over a standard graded $\mathbb{k}$-algebra A. Then $M$ is approximately Cohen-Macaulay if and only if it is sequentially and almost Cohen-Macaulay.

Theorem 2.17. Let $M$ and $N$ be two finitely generated graded modules over standard graded $\mathbb{k}$-algebras $A$ and $B$, respectively. Assume that $M$ is not CohenMacaulay. Then

(1) $M \otimes_{\mathbb{k}} N$ is almost Cohen-Macaulay if and only if $M$ is almost CohenMacaulay and $N$ is Cohen-Macaulay.

(2) $M \otimes_{\mathbb{k}} N$ is approximately Cohen-Macaulay if and only if $M$ is approximately Cohen-Macaulay and $N$ is Cohen-Macaulay.

Proof. To prove statement (1), we assume that $M \otimes_{\mathbb{k}} N$ is almost Cohen-Macaulay. Then by Corollary 2.3, we can write

$$
0 \leq\left(\operatorname{dim}_{A} M-\operatorname{depth}_{A} M\right)+\left(\operatorname{dim}_{B} N-\operatorname{depth}_{B} N\right) \leq 1 .
$$

This implies that $\operatorname{depth}_{A} M=\operatorname{dim}_{A} M-1$ and $\operatorname{depth}_{B} N=\operatorname{dim}_{B} N$ because $M$ is not Cohen-Macaulay, as required.

The converse statement can be proved similarly, by using Corollary 2.3.

(2) follows from statement (1), Theorem 2.11, and Theorem 2.16.

Remark 2.18. For simplicial complexes, the notions of almost and approximately Cohen-Macaulay have combinatorial characterizations. A simplicial complex $\Delta$ is almost Cohen-Macaulay over a field $\mathbb{k}$ if and only if the codimension one skeleton of $\Delta$ is Cohen-Macauly over $\mathbb{k}$ (cf. [1, Exercise 5.1.22]). Recall that the $r$-skeleton of the simplicial complex $\Delta$ is defined as $\Delta^{r}:=\{\sigma \in \Delta \mid \operatorname{dim} \sigma \leq$ $r$ \}. By Theorem 2.16, an approximately simplicial complex $\Delta$ can be described combinatorially through the several combinatorial characterizations of sequential Cohen-Macaulayness (cf. Remark 2.12).

Example 2.19. (1) Every disconnected simplicial complex of dimension 1 or connected non-pure simplicial complex of dimension 2 is almost CohenMacaulay.

(2) Every connected non-pure shellable simplicial complex of dimension 2 is approximately Cohen-Macaulay.

Corollary 2.20. Let $\Delta$ and $\Delta^{\prime}$ be two simplicial complexes over disjoint vertex sets. Then

(1) $\Delta * \Delta^{\prime}$ is almost Cohen-Macaulay (over $\mathbb{k}$ ) if and only if one of $\Delta$ or $\Delta^{\prime}$ is Cohen-Macaulay (over $\mathbb{k})$ and the other is almost Cohen-Macaulay (over $\mathbb{k}$ ). 
(2) $\Delta * \Delta^{\prime}$ is approximately Cohen-Macaulay (over $\left.\mathbb{k}\right)$ if and only if one of $\Delta$ or $\Delta^{\prime}$ is Cohen-Macaulay (over $\mathbb{k})$ and the other is approximately Cohen-Macaulay (over $\mathbb{k})$.

Example 2.21. Consider the simplicial complex $\Delta=\left\langle\left\{x_{1}, x_{2}, x_{5}\right\},\left\{x_{3}, x_{4}, x_{5}\right\}\right\rangle$ over the vertex set $\left\{x_{1}, \ldots, x_{5}\right\}$. We have $\Delta=\Delta^{\prime} * \Delta^{\prime \prime}$ where $\Delta^{\prime}=\left\langle\left\{x_{1}, x_{2}\right\}\right.$, $\left.\left\{x_{3}, x_{4}\right\}\right\rangle$ is a simplicial complex over $\left\{x_{1}, \ldots, x_{4}\right\}$, and $\Delta^{\prime \prime}=\left\langle\left\{x_{5}\right\}\right\rangle$ is a simplicial complex over $\left\{x_{5}\right\}$. We have $\Delta^{\prime}$ is almost Cohen-Macaulay because it is 1-dimensional and disconnected. Hence, $\Delta$ is almost Cohen-Macaulay because $\Delta "$ is Cohen-Macaulay.

\section{Cleanness}

The notion of cleanness was introduced by Dress in [3] as an algebraic counterpart of the notion of shellability for the simplicial complexes. The aim of this section is to present an algebraic way to show that shellability is preserved under simplicial join.

For a non-zero finitely generated module $M$ over a Noetherian ring $A$, it is well-known (cf. [11, Theorem 6.4]) that there exists a finite prime filtration

$$
\mathcal{F}: 0=M_{0} \subset M_{1} \subset \cdots \subset M_{r-1} \subset M_{r}=M
$$

with the cyclic quotients $M_{i} / M_{i-1} \simeq A / \mathfrak{p}_{i}$ where $\mathfrak{p}_{i} \in \operatorname{Supp}_{A}(M)$. The support of $\mathcal{F}$ is the set of prime ideals $\operatorname{Supp}_{A}(\mathcal{F}):=\left\{\mathfrak{p}_{1}, \ldots, \mathfrak{p}_{r}\right\}$. By [11, Theorem 6.3], we have

$$
\operatorname{Min}_{A}(M) \subseteq \operatorname{Ass}_{A}(M) \subseteq \operatorname{Supp}_{A}(\mathcal{F}) \subseteq \operatorname{Supp}_{A}(M) .
$$

Here, $\operatorname{Supp}_{A}(M), \operatorname{Min}_{A}(M)$, and $\operatorname{Ass}_{A}(M)$ denote the usual support of $M$, the set of minimal primes of $\operatorname{Supp}_{A}(M)$, and the set of associated primes of $M$, respectively.

Definition 3.1 (Dress [3]). A prime filtration $\mathcal{F}$ of a non-zero finitely generated module $M$ over a Noetherian ring $A$ is called clean if $\operatorname{Supp}_{A}(\mathcal{F}) \subseteq \operatorname{Min}_{A}(M)$. The $A$-module $M$ is called clean if it admits a clean filtration.

Remark 3.2. In [8, Lemma 3.1], Herzog and Popescu gave the following useful characterization of a clean filtration: A prime filtration $\mathcal{F}$ of a non-zero finitely generated module $M$ over a Noetherian ring $A$ is clean if and only if for all $i, j$ for which $\mathfrak{p}_{i} \subseteq \mathfrak{p}_{j}$ it follows that $\mathfrak{p}_{i}=\mathfrak{p}_{j}$.

Theorem 3.3. Let $A$ and $B$ be two Noetherian $\mathbb{k}$-algebras such that $A \otimes_{\mathbb{k}} B$ is Noetherian. Let $M$ and $N$ be two finitely generated modules over $A$ and $B$, respectively. Assume that $A / \mathfrak{p} \otimes_{\mathbb{k}} B / \mathfrak{q}$ is an integral domain for all $\mathfrak{p} \in \operatorname{Ass}_{A}(M)$, and $\mathfrak{q} \in \operatorname{Ass}_{B}(N)$. If $M$ and $N$ are clean, then $M \otimes_{\mathbb{k}} N$ is clean.

Remark 3.4. In this paper, we are interested in the case that $A$ and $B$ are polynomial rings over the field $\mathbb{k}$, and $M=A / I$ and $N=B / J$ where $I$ and $J$ are monomial ideals. In this case, $A / \mathfrak{p} \otimes_{\mathbb{k}} B / \mathfrak{q}$ is an integral domain for all $\mathfrak{p} \in \operatorname{Ass}_{A}(M)$, and $\mathfrak{q} \in \operatorname{Ass}_{B}(N)$ because the prime ideals are generated by variables. However, there are other cases for satisfying the hypothesis. For instance, we can consider the case that the field $\mathbb{k}$ is algebraically closed (cf. [5, Exercise A1.2]). 
In the sequel, we provide the requirements for the proof of Theorem 3.3.

Lemma 3.5. Let $A$ and $B$ be two $\mathbb{k}$-algebras. Let $I, I^{\prime}$ be ideals of $A$, and $J, J^{\prime}$ ideals of $B$ such that $I \otimes_{\mathbb{k}} B+A \otimes_{\mathbb{k}} J \subseteq I^{\prime} \otimes_{\mathbb{k}} B+A \otimes_{\mathbb{k}} J^{\prime}$. Then $I \subseteq I^{\prime}$ and $J \subseteq J^{\prime}$.

Proof. We may assume that $I^{\prime} \neq A$ and $J^{\prime} \neq B$. We consider the diagram

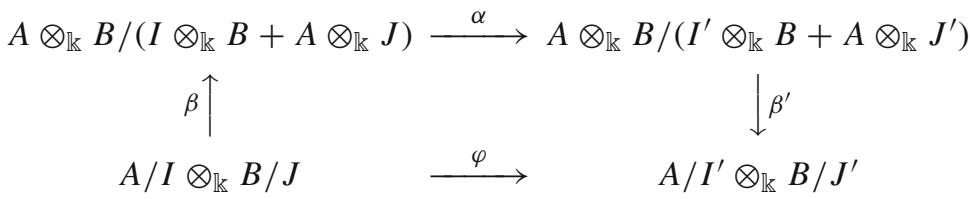

in which $\alpha$ is a natural ring epimorphism, $\beta, \beta^{\prime}$ are ring isomorphisms defined by

$$
\begin{aligned}
& \beta((a+I) \otimes(b+J))=(a \otimes b)+I \otimes_{\mathbb{k}} B+A \otimes_{\mathbb{k}} J, \\
& \beta^{\prime}\left((a \otimes b)+I^{\prime} \otimes B+A \otimes J^{\prime}\right)=\left(a+I^{\prime}\right) \otimes\left(b+J^{\prime}\right),
\end{aligned}
$$

and $\varphi=\beta^{\prime} \circ \alpha \circ \beta$. We can check that $\varphi((a+I) \otimes(b+J))=\left(a+I^{\prime}\right) \otimes\left(b+J^{\prime}\right)$. Now, we consider an arbitrary element $x \in I$. Then for an element $y \notin J^{\prime}$, we have

$$
0=\varphi((x+I) \otimes(y+J))=\left(x+I^{\prime}\right) \otimes\left(y+J^{\prime}\right),
$$

which implies $x \in I^{\prime}$. Similarly, we have $J \subseteq J^{\prime}$.

Lemma 3.6. Let $\varphi: A \rightarrow B$ be a homomorphism of Noetherian rings. Let $M$ be a finitely generated $A$-module, and $N$ a finitely generated $B$-module which is flat over A by means of $\varphi$. Assume that $M \otimes_{A} N \neq 0$. Then for each prime filtration $\mathcal{F}_{M}$ of the A-module $M$, there exist a prime filtration $\mathcal{F}_{M \otimes_{A} N}$ of the $B$-module $M \otimes_{A} N$, and a prime filtration $\mathcal{F}_{N / \mathfrak{p} N}$ of the $B$-module $N / \mathfrak{p} N$ for each $\mathfrak{p} \in \operatorname{Supp}_{A} \mathcal{F}_{M}$ with $N \neq \mathfrak{p} N$ such that

$$
\operatorname{Supp}_{B} \mathcal{F}_{M \otimes_{A} N}=\bigcup_{\substack{\mathfrak{p} \in \operatorname{Supp}_{A} \\ N \neq \mathfrak{p} N}} \mathcal{F}_{M} \operatorname{Supp}_{B} \mathcal{F}_{N / \mathfrak{p} N}
$$

Proof. We assume that

$$
\mathcal{F}_{M}: 0=M_{0} \subset M_{1} \subset \cdots \subset M_{r}=M
$$

with cyclic quotients $M_{i} / M_{i-1} \simeq A / \mathfrak{p}_{i}$ with $\mathfrak{p}_{i} \in \operatorname{Supp}_{A}(M)$. We consider the filtration

$$
0=M_{0} \otimes_{A} N \subseteq M_{1} \otimes_{A} N \subseteq \cdots \subseteq M_{r} \otimes_{A} N=M \otimes_{A} N
$$

We have

$$
\left(M_{i} \otimes_{A} N\right) /\left(M_{i-1} \otimes_{A} N\right) \simeq\left(M_{i} / M_{i-1}\right) \otimes_{A} N \simeq N / \mathfrak{p}_{i} N
$$


in which the first isomorphism holds because of $A$-flatness of $N$. Hence $M_{i} \otimes_{A} N=$ $M_{i-1} \otimes_{A} N$ precisely when $N=\mathfrak{p}_{i} N$. Let $\left\{i_{0}, \ldots, i_{\ell}\right\}$ be a subset of $\{0,1, \ldots, r\}$ for which we have

$$
0=M_{i_{0}} \otimes_{A} N \subset M_{i_{1}} \otimes_{A} N \subset \cdots \subset M_{i_{\ell}} \otimes_{A} N=M \otimes_{A} N
$$

We assume that $\mathcal{F}_{j}$ is a prime filtration of the $B$-module $\left(M_{i_{j}} \otimes_{A} N\right) /\left(M_{i_{j-1}}\right.$ $\left.\otimes_{A} N\right) \simeq N / \mathfrak{p}_{i_{j}} N$, for each $j=1, \ldots, \ell$, and we regard it as

$$
\mathcal{F}_{j}: M_{i_{j-1}} \otimes_{A} N=L_{j, 0} \subset L_{j, 1} \subset \cdots \subset L_{j, s_{j}}=M_{i_{j}} \otimes_{A} N
$$

We can refine the filtration (*) to a prime filtration $\mathcal{F}_{M \otimes_{A} N}$ of the $B$-module $M \otimes_{A} N$ by inserting the filtration $\mathcal{F}_{j}$ in it for all $j=1, \ldots, \ell$. Using this observation, we can conclude the result.

Corollary 3.7. Let $A$ and $B$ be two Noetherian $\mathbb{k}$-algebras such that $A \otimes_{\mathbb{k}} B$ is Noetherian. Let $M$ and $N$ be two non-zero modules over $A$ and $B$, respectively. Then

(1) $\operatorname{Ass}_{A \otimes_{\mathbb{k}} B}\left(M \otimes_{\mathbb{k}} N\right)=\bigcup_{\mathfrak{p} \in \operatorname{Ass}_{A}(M)} \bigcup_{\mathfrak{q} \in \operatorname{Ass}_{B}(N)} \operatorname{Ass}_{A \otimes_{\mathbb{R}} B}\left(A / \mathfrak{p} \otimes_{\mathbb{R}} B / \mathfrak{q}\right)$.

(2) Assume in addition that the A-module $M$ and the $B$-module $N$ are finitely generated modules with prime filtration $\mathcal{F}_{M}$ and $\mathcal{F}_{N}$, respectively. Then there exist a prime filtration $\mathcal{F}_{M \otimes_{\mathbb{R}} N}$ of the $A \otimes_{\mathbb{k}} B$-module $M \otimes_{\mathbb{k}} N$ and a prime filtration $\mathcal{F}_{A / \mathfrak{p} \otimes_{\mathbb{k}} B / \mathfrak{q}}$ of the $A \otimes_{\mathbb{k}} B$-module $A / \mathfrak{p} \otimes_{\mathbb{k}} B / \mathfrak{q}$ for each prime $\mathfrak{p} \in$ $\operatorname{Supp}_{A} \mathcal{F}_{M}$ and $\mathfrak{q} \in \operatorname{Supp}_{B} \mathcal{F}_{N}$ such that

$$
\operatorname{Supp}_{A \otimes_{\mathbb{k}} B} \mathcal{F}_{M \otimes_{\mathbb{k}} N}=\bigcup_{\mathfrak{p} \in \operatorname{Supp}_{A} \mathcal{F}_{M}} \bigcup_{\mathfrak{q} \in \operatorname{Supp}_{B} \mathcal{F}_{N}} \operatorname{Supp}_{A \otimes_{\mathbb{k}} B} \mathcal{F}_{A / \mathfrak{p} \otimes_{\mathbb{k}} B / \mathfrak{q}} .
$$

Proof. Both statements can be proved by the same technique and by using [11, Theorem 23.2(ii)] and Lemma 3.6, respectively. We only prove (2).

The $A \otimes_{\mathbb{k}} B$-module $A \otimes_{\mathbb{k}} N$ is $A$-flat by means of the homomorphism $A \rightarrow$ $A \otimes_{\mathbb{k}} B$. Since $M \otimes_{\mathbb{k}} N \simeq M \otimes_{A}\left(A \otimes_{\mathbb{k}} N\right)$, we can apply Lemma 3.6 to write

$$
\begin{aligned}
\operatorname{Supp}_{A \otimes_{\mathbb{k}} B} \mathcal{F}_{M \otimes_{\mathbb{k}} N} & =\operatorname{Supp}_{A \otimes_{\mathbb{k}} B} \mathcal{F}_{M \otimes_{A}\left(A \otimes_{\mathbb{k}} N\right)} \\
& =\bigcup_{\mathfrak{p} \in \operatorname{Supp}_{A} \mathcal{F}_{M}} \operatorname{Supp}_{A \otimes_{\mathbb{k}} B} \mathcal{F}_{A / \mathfrak{p} \otimes_{A}\left(A \otimes_{\mathbb{k}} N\right)}
\end{aligned}
$$

Now the $A \otimes_{\mathbb{k}} B$-module $A / \mathfrak{p} \otimes_{\mathbb{k}} B$ is flat over $B$ by means of the homomorphism $B \rightarrow A \otimes_{\mathbb{k}} B$ of Noetherian rings. Hence we can use the isomorphism $A / \mathfrak{p} \otimes_{\mathbb{k}} N \simeq$ $\left(A / \mathfrak{p} \otimes_{\mathbb{k}} B\right) \otimes_{B} N$ along with Lemma 3.6 to write

$$
\operatorname{Supp}_{A \otimes_{\mathbb{k}} B} \mathcal{F}_{N \otimes_{B}\left(A / \mathfrak{p} \otimes_{\mathbb{k}} B\right)}=\bigcup_{\mathfrak{q} \in \operatorname{Supp}_{B} \mathcal{F}_{N}} \operatorname{Supp}_{A \otimes_{\mathbb{k}} B} \mathcal{F}_{\left(A / \mathfrak{p} \otimes_{\mathbb{k}} B\right) \otimes_{B} B / \mathfrak{q}}
$$

which implies the result. 
Proof of Theorem 3.3. Let $\mathcal{F}_{M}$ and $\mathcal{F}_{N}$ be the clean filtration of the $A$-module $M$ and the $B$-module $N$, respectively. By Lemma 3.6, there exists a prime filtration $\mathcal{F}_{M \otimes_{\mathbb{k}} N}$ of $A \otimes_{\mathbb{k}} B$-module $M \otimes_{\mathbb{k}} N$, and a prime filtration $\mathcal{F}_{A / \mathfrak{p} \otimes_{\mathbb{k}} B / \mathfrak{q}}$ for each $\mathfrak{p} \in \operatorname{Supp}_{A} \mathcal{F}_{M}$ and $\mathfrak{q} \in \operatorname{Supp}_{B} \mathcal{F}_{N}$ such that

$$
\operatorname{Supp}_{A \otimes_{\mathbb{R}} B} \mathcal{F}_{M \otimes_{\mathbb{R}} N}=\bigcup_{\mathfrak{p} \in \operatorname{Supp}_{A}} \bigcup_{\mathcal{F}_{M}} \bigcup_{\mathfrak{q} \in \operatorname{Supp}_{B} \mathcal{F}_{N}} \operatorname{Supp}_{A \otimes_{\mathbb{k}} B} \mathcal{F}_{A / \mathfrak{p} \otimes_{\mathbb{k}} B / \mathfrak{q}} .
$$

Since $\mathcal{F}_{M}$ and $\mathcal{F}_{N}$ are clean, we have that

$$
\operatorname{Supp}_{A} \mathcal{F}_{M}=\operatorname{Ass}_{A}(M) \text {, and } \operatorname{Supp}_{B} \mathcal{F}_{N}=\operatorname{Ass}_{B}(N) \text {. }
$$

On the other hand, our hypothesis implies that

$$
\operatorname{Supp}_{A \otimes_{\mathbb{k}} B} \mathcal{F}_{A / \mathfrak{p} \otimes_{\mathbb{k}} B / \mathfrak{q}}=\operatorname{Ass}_{A \otimes_{\mathbb{k}} B}\left(A / \mathfrak{p} \otimes_{\mathbb{k}} B / \mathfrak{q}\right) .
$$

Combining these relations with Corollary 3.7, we see that

$$
\begin{aligned}
\operatorname{Supp}_{A \otimes_{\mathbb{k}} B} \mathcal{F}_{M \otimes_{\mathbb{k}} N} & =\operatorname{Ass}_{A \otimes_{\mathbb{k}} B}\left(M \otimes_{\mathbb{k}} N\right) \\
& =\bigcup_{\mathfrak{p} \in \operatorname{Ass}_{A}(M)} \bigcup_{\mathfrak{q} \in \operatorname{Ass}_{B}(N)}\left\{\mathfrak{p} \otimes_{\mathbb{k}} B+A \otimes_{\mathbb{k}} \mathfrak{q}\right\} .
\end{aligned}
$$

If we assume that $\mathfrak{p} \otimes_{\mathbb{k}} B+A \otimes_{\mathbb{k}} \mathfrak{q} \subseteq \mathfrak{p}^{\prime} \otimes_{\mathbb{k}} B+A \otimes_{\mathbb{k}} \mathfrak{q}^{\prime}$ for $\mathfrak{p}, \mathfrak{p}^{\prime} \in \operatorname{Ass}_{A}(M)$ and $\mathfrak{q}, \mathfrak{q}^{\prime} \in \operatorname{Ass}_{B}(N)$, then we have $\mathfrak{p} \subseteq \mathfrak{p}^{\prime}$ and $\mathfrak{q} \subseteq \mathfrak{q}^{\prime}$, by Lemma 3.5. Therefore by cleanness of $M$ and $N$, we get the result.

We are now ready to state and prove the main result of this section.

Corollary 3.8. Let $I$ and $J$ be two arbitrary monomial ideals in the polynomial rings $R=\mathbb{k}\left[x_{1}, \ldots, x_{n}\right]$ and $S=\mathbb{k}\left[y_{1}, \ldots, y_{m}\right]$, respectively. Then $R / I \otimes_{\mathbb{k}} S / J$ is clean if and only if $R / I$ and $S / J$ are clean.

Proof. If $R / I$ and $S / J$ are clean, then we can apply Theorem 3.3 to get the result.

Conversely, let $T=R \otimes_{\mathbb{k}} S$, and

$$
\mathcal{F}_{T /(I+J)}: I+J=L_{0} \subset L_{1} \subset \cdots \subset L_{r-1} \subset L_{r}=T
$$

be a clean filtration of $T /(I+J)$ such that

(1) $L_{i}=\left(L_{i-1}, u_{i}\right)$ where $u_{i}$ is a monomial in $T$; and

(2) $L_{i} / L_{i-1} \simeq T / \mathfrak{P}_{i}$ where $\mathfrak{P}_{i}=\left(L_{i-1}: u_{i}\right)$ is a monomial prime ideal of $T$.

We have $\mathfrak{P}_{i} \in \operatorname{Ass}(T /(I+J))$ because $\mathcal{F}_{T /(I+J)}$ is clean. Setting $I_{i}:=L_{i} \cap R$, we get the filtration

$$
I_{0} \subseteq I_{1} \subseteq \cdots \subseteq I_{r}=R
$$

We have $I_{0}=I$, and $I_{i}=I_{i-1}$ precisely when $u_{i} \notin R$. Let $\left\{i_{0}, \ldots, i_{\ell}\right\}$ be the subset of $\{0, \ldots, r\}$ for which we have $I_{i_{j}}$ is properly contained in $I_{i_{j+1}}$ in the filtration

$$
I=I_{i_{0}} \subset I_{i_{1}} \subset \cdots \subset I_{i_{\ell}}=R
$$


Then we have

$$
\begin{aligned}
I_{i_{j}} / I_{i_{j-1}} & =\left(I_{i_{j-1}}, u_{i_{j}}\right) / I_{i_{j-1}} \\
& \simeq R /\left(I_{i_{j-1}}:_{R} u_{i_{j}}\right) \\
& =R /\left(L_{i_{j-1}}:_{T} u_{i_{j}}\right) \cap R \\
& =R /\left(\mathfrak{P}_{i_{j}} \cap R\right) .
\end{aligned}
$$

We set $\mathfrak{p}_{i_{j}}:=\mathfrak{P}_{i_{j}} \cap R$. For all $i_{j}, i_{k}$ for which we have $\mathfrak{p}_{i_{j}} \subseteq \mathfrak{p}_{i_{k}}$, we can consider the inclusion $\mathfrak{p}_{i_{j}}+\mathfrak{q} \subseteq \mathfrak{p}_{i_{k}}+\mathfrak{q}$ in $\operatorname{Ass}(T /(I+J))=\operatorname{Supp} \mathcal{F}_{T /(I+J)}$ where $\mathfrak{q} \in \operatorname{Ass}_{S}(S / J)$. Since $\mathcal{F}_{T /(I+J)}$ is clean, we conclude that $\mathfrak{p}_{i_{j}}=\mathfrak{p}_{i_{k}}$, as required.

\section{Pretty cleanness}

The aim of this section is to present an algebraic way to show that the notion of shellability for the simplicial multicomplexes introduced by Herzog and Popescu [8] is preserved under simplicial join of multicomplexes. Here we recall some basic definitions and results related to multicomplexes, and we refer the reader to [8, Sect. 9] for more details.

For a subset $\Gamma \subseteq \mathbb{N}_{\infty}^{n}$ where $\mathbb{N}_{\infty}:=\mathbb{N} \cup\{\infty\}$ with $a<\infty$ for all $a \in \mathbb{N}$, the set of all maximal elements of $\Gamma$ with respect to the componentwise partial order $\leq$ is denoted by $\mathcal{M}(\Gamma)$. The subset $\Gamma$ is called a multicomplex if it is closed under going down, and for each element $\mathbf{a} \in \Gamma$, there exists $\mathbf{m} \in \mathcal{M}(\Gamma)$ with $\mathbf{a} \leq \mathbf{m}$.

For each multicomplex $\Gamma \subseteq \mathbb{N}_{\infty}^{n}$, the $\mathbb{k}$-subspace in $R=\mathbb{k}\left[x_{1}, \ldots, x_{n}\right]$ spanned by all monomials $x_{1}^{a_{1}} \ldots x_{n}^{a_{n}}$ with $\left(a_{1}, \ldots, a_{n}\right) \notin \Gamma$ is a monomial ideal denoted by $I_{\Gamma}$. The correspondence $\Gamma \rightsquigarrow \mathbb{k}[\Gamma]:=R / I_{\Gamma}$ constitutes a bijective from simplicial multicomplexes $\Gamma$ in $\mathbb{N}_{\infty}^{n}$ to monomial ideals inside $R$.

Let $\Gamma^{\prime} \subseteq \mathbb{N}_{\infty}^{m}$ be a second simplicial multicomplex. The simplicial join $\Gamma$ with $\Gamma^{\prime}$ is the simplicial multicomplex

$$
\Gamma * \Gamma^{\prime}:=\left\{\overline{\mathbf{a}}+\overline{\mathbf{b}} \mid \mathbf{a} \in \Gamma \text { and } \mathbf{b} \in \Gamma^{\prime}\right\} \subseteq \mathbb{N}_{\infty}^{n+m},
$$

where ${ }^{-}: \mathbb{N}_{\infty}^{n} \rightarrow \mathbb{N}_{\infty}^{n+m}$ and ${ }^{-}: \mathbb{N}_{\infty}^{m} \rightarrow \mathbb{N}_{\infty}^{n+m}$ are canonical embedding defined by $\overline{\mathbf{a}}:=\left(a_{1}, \ldots, a_{n}, 0, \ldots, 0\right)$ and $\overline{\mathbf{b}}:=\left(0, \ldots, 0, b_{1}, \ldots, b_{m}\right)$ where $\mathbf{a}:=\left(a_{1}, \ldots, a_{n}\right)$ and $\mathbf{b}:=\left(b_{1}, \ldots, b_{m}\right)$. As in the case of simplicial complexes, we have the (graded) $\mathbb{k}$-algebra isomorphism $\mathbb{k}\left[\Gamma * \Gamma^{\prime}\right] \simeq \mathbb{k}[\Gamma] \otimes_{\mathbb{k}} \mathbb{k}\left[\Gamma^{\prime}\right]$.

A multicomplex $\Gamma$ is shellable in the sense of [8, Definition 10.2] if and only if the $\mathbb{k}$-algebra $\mathbb{k}[\Gamma]$ is pretty clean in the following sense.

Definition 4.1 (Herzog and Popescu [8]). A prime filtration

$$
\mathcal{F}: 0=M_{0} \subset M_{1} \subset \cdots \subset M_{r-1} \subset M_{r}=M
$$

of a non-zero finitely generated module $M$ over a Noetherian ring $A$ with the cyclic quotients $M_{i} / M_{i-1} \simeq A / \mathfrak{p}_{i}$ is called pretty clean if for all $i<j$ for which $\mathfrak{p}_{i} \subseteq \mathfrak{p}_{j}$ it follows that $\mathfrak{p}_{i}=\mathfrak{p}_{j}$. The module $M$ is called pretty clean if it admits a pretty clean filtration. 
To prove our main result we use the concept of dimension filtration which is originally due to Schenzel [14].

Definition 4.2. Let $M$ be a finitely generated module over a Noetherian ring $A$ with $\operatorname{dim}(M)=d$. The filtration

$$
0 \subseteq Q_{A}^{0}(M) \subseteq Q_{A}^{1}(M) \subseteq \cdots \subseteq Q_{A}^{d}(M)=M,
$$

which is defined by the property that $Q_{A}^{i}(M)$ is the largest submodule of $M$ with $\operatorname{dim} Q_{A}^{i}(M) \leq i$ is called the dimension filtration of $M$.

Remark 4.3. Let $\operatorname{Ass}_{A}^{i}(M):=\left\{\mathfrak{p} \in \operatorname{Ass}_{A}(M) \mid \operatorname{dim}(A / \mathfrak{p})>i\right\}$ for $i=0, \ldots, d$. Then by [14, Proposition 2.2], we have $Q_{A}^{i}(M)=H_{\mathfrak{A}_{A}^{i}}^{0}(M)$ where $\mathfrak{A}_{A}^{i}$ is the product of all $\mathfrak{p} \in \operatorname{Ass}_{A}(M) \backslash \operatorname{Ass}_{A}^{i}(M)$ with the convention that $\mathfrak{A}_{A}^{i}=A$ if $\operatorname{Ass}_{A}(M) \backslash \operatorname{Ass}_{A}^{i}(M)$ is empty. Consequently, when $M$ is a graded module, then all modules $Q_{A}^{i}(M)$ are graded.

Remark 4.4. It is obvious that if $\operatorname{dim} Q_{A}^{i+1}(M) \leq i$, then $Q_{A}^{i}(M)=Q_{A}^{i+1}(M)$. Hence, we can describe the dimension filtration of the $A$-module $M$ as the filtration

$$
0=M_{0} \subset M_{1} \subset \cdots \subset M_{t-1} \subset M_{t}=M
$$

of $M$ in which $M_{i-1}$ is the largest submodule of $M_{i}$ which has dimension strictly less than $\operatorname{dim} M_{i}$ for all $i$. Moreover, for $i=1, \ldots, t$ we have $M_{i}=Q_{A}^{d_{i}}(M)$ where $d_{i}=\operatorname{dim} M_{i}$. In the remainder of this section, we use this description of a dimension filtration.

Lemma 4.5. Let $\varphi:(A, \mathfrak{m}) \rightarrow(B, \mathfrak{n})$ be a homomorphism of standard graded $\mathbb{k}$-algebras with $\varphi(\mathfrak{m}) \subseteq \mathfrak{n}$. Let $M$ be a non-zero finitely generated graded $A$-module, and $N$ a non-zero finitely generated graded $B$-module which is flat over $A$ by means of $\varphi$. Assume that for each $\mathfrak{p} \in \operatorname{Ass}_{A}^{k}(M)$ and $k=0, \ldots, \operatorname{dim} M-1$, we have

$$
\operatorname{Ass}_{B}(N / \mathfrak{p} N)=\operatorname{Ass}_{B}^{k+\ell}(N / \mathfrak{p} N),
$$

where $\ell=\operatorname{dim}_{B}(N / \mathfrak{m} N)$. If

$$
0=M_{0} \subset M_{1} \subset \cdots \subset M_{t}=M
$$

is the dimension filtration of the A-module $M$, then

$$
0=M_{0} \otimes_{A} N \subset M_{1} \otimes_{A} N \subset \cdots \subset M_{t} \otimes_{A} N=M \otimes_{A} N
$$

is the dimension filtration of the $B$-module $M \otimes_{A} N$.

Proof. It follows from the equality $Q_{A}^{k}(M) \otimes_{A} N=Q_{B}^{k+\ell}\left(M \otimes_{A} N\right)$ which can be proved by an argument similar to [18, Proposition 3]. 
Lemma 4.6. Let $M$ and $N$ be two finitely generated graded modules over standard graded algebras $(A, \mathfrak{m})$ and $(B, \mathfrak{n})$, respectively. Assume $A / \mathfrak{p} \otimes_{\mathbb{k}} B / \mathfrak{q}$ is an integral domain for all $\mathfrak{p} \in \operatorname{Ass}_{A}(M)$ and $\mathfrak{q} \in \operatorname{Ass}_{B}(N)$. Assume in addition that

$$
\text { (0) }=M_{0} \subset M_{1} \subset \cdots \subset M_{s}=M
$$

and

$$
\text { (0) }=N_{0} \subset N_{1} \subset \cdots \subset N_{t}=N
$$

are the dimension filtration of $M$ and $N$. Then there exists the dimension filtration

$$
\cdots \subset M_{i-1} \otimes_{\mathbb{k}} N=L_{i, 0} \subset L_{i, 1} \subset \cdots \subset L_{i, t}=M_{i} \otimes_{\mathbb{k}} N \subset \cdots
$$

of the $A \otimes_{\mathbb{k}} B$-module $M \otimes_{\mathbb{k}} N$ such that

$$
L_{i, j} / L_{i, j-1} \simeq\left(M_{i} / M_{i-1}\right) \otimes_{\mathbb{k}}\left(N_{j} / N_{j-1}\right)
$$

for all $i$ and $j$.

Proof. We show that the filtration

$$
(0)=M_{0} \otimes_{\mathbb{k}} N \subset \cdots \subset M_{s} \otimes_{\mathbb{k}} N=M \otimes_{\mathbb{k}} N
$$

can be refined to the desired dimension filtration of the $A \otimes_{\mathbb{k}} B$-module $M \otimes_{\mathbb{k}} N$. To this end, first we claim that for each factor $M_{i} / M_{i-1}$, the filtration

$$
(0)=\left(M_{i} / M_{i-1}\right) \otimes_{\mathbb{k}} N_{0} \subset \cdots \subset\left(M_{i} / M_{i-1}\right) \otimes_{\mathbb{k}} N_{t}=\left(M_{i} / M_{i-1}\right) \otimes_{\mathbb{k}} N
$$

is the dimension filtration of $\left(M_{i} / M_{i-1}\right) \otimes_{\mathbb{k}} N$.

To prove the claim, we notice that the $B$-module $N$ is finitely generated, and the $A \otimes_{\mathbb{k}} B$-module $\left(M_{i} / M_{i-1}\right) \otimes_{\mathbb{k}} B$ is finitely generated and $B$-flat by means of the homomorphism $B \rightarrow A \otimes_{\mathbb{k}} B$. Hence, by Lemma 4.5, we need only to prove that for each $\mathfrak{q} \in \operatorname{Ass}_{B}^{k}(N)$ with $0 \leq k \leq \operatorname{dim}(N)-1$,

$$
\operatorname{Ass}_{A \otimes_{\mathbb{k}} B}\left(B / \mathfrak{q} \otimes_{\mathbb{k}} M_{i} / M_{i-1}\right)=\operatorname{Ass}_{A \otimes_{\mathbb{k}} B}^{k+\ell}\left(B / \mathfrak{q} \otimes_{\mathbb{k}} M_{i} / M_{i-1}\right),
$$

where $\ell=\operatorname{dim}_{A \otimes_{\mathbb{k}} B}\left(B / \mathfrak{n} \otimes_{\mathbb{k}} M_{i} / M_{i-1}\right)$.

For an arbitrary element $\mathfrak{P} \in \operatorname{Ass}_{A \otimes_{\mathbb{k}} B}\left(B / \mathfrak{q} \otimes_{\mathbb{k}} M_{i} / M_{i-1}\right)$, there exists an element $\mathfrak{p} \in \operatorname{Ass}_{A}\left(M_{i} / M_{i-1}\right)$ such that $\mathfrak{P} \in \operatorname{Ass}_{A \otimes_{\mathbb{k}} B}\left(B / \mathfrak{q} \otimes_{\mathbb{k}} A / \mathfrak{p}\right)$, by Corollary 3.7. Hence our hypothesis implies that $\mathfrak{P}=\mathfrak{p} \otimes_{\mathbb{k}} B+A \otimes_{\mathbb{k}} \mathfrak{q}$, and so

$$
\begin{aligned}
\operatorname{dim}\left(\left(A \otimes_{\mathbb{k}} B\right) / \mathfrak{P}\right) & =\operatorname{dim}\left(B / \mathfrak{q} \otimes_{\mathbb{k}} A / \mathfrak{p}\right) \\
& =\operatorname{dim} B / \mathfrak{q}+\operatorname{dim} A / \mathfrak{p} \\
& >k+\operatorname{dim} A / \mathfrak{p} .
\end{aligned}
$$

Since $M_{i-1}$ is the largest graded submodule of $M_{i}$ of dimension strictly less than $\operatorname{dim} M_{i}$, we can see that $\operatorname{dim} A / \mathfrak{p}=\operatorname{dim}\left(M_{i} / M_{i-1}\right)$. Hence using the equality

$$
\operatorname{dim}_{A}\left(M_{i} / M_{i-1}\right)=\operatorname{dim}_{A \otimes_{\mathbb{k}} B}\left(B / \mathfrak{n} \otimes_{\mathbb{k}} M_{i} / M_{i-1}\right)=\ell,
$$

we conclude that $\operatorname{dim}\left(\left(A \otimes_{\mathbb{k}} B\right) / \mathfrak{P}\right)>k+\ell$ which proves our claim. 
Now, the above claim along with the isomorphism

$$
\left(M_{i} / M_{i-1}\right) \otimes_{\mathbb{k}} N \simeq\left(M_{i} \otimes_{\mathbb{k}} N\right) /\left(M_{i-1} \otimes_{\mathbb{k}} N\right)
$$

imply that there exists a dimension filtration

$$
(0)=L_{i, 0} /\left(M_{i-1} \otimes N\right) \subset \cdots \subset L_{i, t} /\left(M_{i-1} \otimes N\right)
$$

for $A \otimes_{\mathbb{k}} B$-module $\left(M_{i} \otimes N\right) /\left(M_{i-1} \otimes N\right)$ for which we have

$$
L_{i, j} / L_{i, j-1} \simeq\left(M_{i} / M_{i-1}\right) \otimes_{\mathbb{k}}\left(N_{j} / N_{j-1}\right) .
$$

Finally, we notice that $L_{i, j-1}$ is the largest submodule of $L_{i, j}$ which has dimension strictly less than $\operatorname{dim}_{A \otimes_{\mathbb{k}} B}\left(L_{i, j}\right)$ because $L_{i, j-1} /\left(M_{i-1} \otimes_{\mathbb{k}} N\right)$ is the largest submodule of $L_{i, j} /\left(M_{i-1} \otimes_{\mathbb{k}} N\right)$ whose dimension is strictly less than $\operatorname{dim}_{A \otimes_{\mathbb{R}} B}\left(L_{i, j} /\left(M_{i-1} \otimes N\right)\right)$.

Theorem 4.7. Let $M$ and $N$ be two finitely generated graded modules over standard graded Cohen-Macaulay $\mathbb{k}$-algebras $(A, \mathfrak{m})$ and $(B, \mathfrak{n})$ with canonical modules $\omega_{A}$ and $\omega_{B}$, respectively. Assume that $A / \mathfrak{p} \otimes_{\mathbb{k}} B / \mathfrak{q}$ is an integral domain for all $\mathfrak{p} \in \operatorname{Ass}_{A}(M)$, and $\mathfrak{q} \in \operatorname{Ass}_{B}(N)$. If $M$ and $N$ are pretty clean modules with pretty clean filtration $\mathcal{F}_{M}$ and $\mathcal{F}_{N}$ such that $A / \mathfrak{p}$ and $B / \mathfrak{q}$ are Cohen-Macaulay for all $\mathfrak{p} \in \operatorname{Supp}\left(\mathcal{F}_{M}\right)$ and $\mathfrak{q} \in \operatorname{Supp}\left(\mathcal{F}_{N}\right)$, then $M \otimes_{\mathbb{k}} N$ is pretty clean.

Proof. The non-zero factors of the dimension filtration of $M$ and $N$ are clean, by [8, Corollary 4.2]. Thus, the non-zero factors of the dimension filtration of $M \otimes_{\mathbb{k}}$ $N$ are clean, by Lemma 4.6, and Theorem 3.3. Hence, the result follows from [8, Corollary 4.2].

As a consequence of Theorem 4.7 we have the following main result of this section.

Corollary 4.8. Let $I$ and $J$ be two arbitrary monomial ideals in the polynomial rings $R=\mathbb{k}\left[x_{1}, \ldots, x_{n}\right]$ and $S=\mathbb{k}\left[y_{1}, \ldots, y_{m}\right]$, respectively. Then $R / I \otimes_{\mathbb{k}} S / J$ is pretty clean if and only if $R / I$ and $S / J$ are pretty clean.

Proof. If $R / I$ and $S / J$ are pretty clean, then Theorem 4.7 gives the result. The converse can be proved by the same argument as Corollary 3.8, and using [8, Corollary 3.6].

Although Theorem 4.7 implies our main result, it is not quite satisfactory because it needs a lot of hypotheses. However, in some special cases like the following, we can reduce these assumptions.

Theorem 4.9. Let $A$ and $B$ be two Noetherian $\mathbb{k}$-algebras such that $A \otimes_{\mathbb{k}} B$ is Noetherian. Let $M$ and $N$ be two finitely generated modules over $A$ and $B$, respectively. Assume that $A / \mathfrak{p} \otimes_{\mathbb{k}} B / \mathfrak{q}$ is an integral domain for all $\mathfrak{p} \in \operatorname{Ass}_{A}(M)$, and $\mathfrak{q} \in \operatorname{Ass}_{B}(N)$. If $M$ is pretty clean and $N$ is clean, then $M \otimes_{\mathbb{k}} N$ is pretty clean. 
Proof. Let

$$
0=M_{0} \subset M_{1} \subset \cdots \subset M_{r-1} \subset M_{r}=M
$$

be the pretty clean filtration of the $A$-module $M$ with the cyclic quotients $M_{i} / M_{i-1} \simeq A / \mathfrak{p}_{i}$. We consider the filtration

$$
0=M_{0} \otimes_{\mathbb{k}} N \subset M_{1} \otimes_{\mathbb{k}} N \subset \cdots \subset M_{r-1} \otimes_{\mathbb{k}} N \subset M_{r} \otimes_{\mathbb{k}} N=M \otimes_{\mathbb{k}} N
$$

of $A \otimes_{\mathbb{k}} B$-modules. Since $A / \mathfrak{p}_{i}$ is a clean $A$-module and $N$ is a clean $B$-module, by Theorem 3.3, the $A \otimes_{\mathbb{k}} B$-module

$$
\left(M_{i} \otimes_{\mathbb{k}} N\right) /\left(M_{i-1} \otimes_{\mathbb{k}} N\right) \simeq\left(M_{i} / M_{i-1}\right) \otimes_{\mathbb{k}} N \simeq\left(A / \mathfrak{p}_{i}\right) \otimes_{\mathbb{k}} N
$$

is clean. For $i=1, \ldots, r$, we assume that $\mathcal{F}_{i}$ is a clean filtration of the $A \otimes_{\mathbb{k}}$ $B$-module $\left(M_{i} \otimes_{\mathbb{k}} N\right) /\left(M_{i-1} \otimes_{\mathbb{k}} N\right)$, and we regard it as

$$
\mathcal{F}_{i}:\left(M_{i-1} \otimes_{\mathbb{k}} N\right)=L_{i, 0} \subset L_{i, 1} \subset \cdots \subset L_{i, s_{i}}=\left(M_{i} \otimes_{\mathbb{k}} N\right)
$$

We have

$$
\begin{aligned}
\operatorname{Supp}_{A \otimes_{\mathbb{k}} B} \mathcal{F}_{i} & =\operatorname{Ass}_{A \otimes_{\mathbb{k}} B}\left(N / \mathfrak{p}_{i} N\right) \\
& =\left\{\mathfrak{p}_{i} \otimes B+A \otimes_{\mathbb{k}} \mathfrak{q}_{i_{j}} \mid \mathfrak{q}_{i_{j}} \in \operatorname{Ass}_{B}(N)\right\},
\end{aligned}
$$

where the first equality is by the cleanness of $N / \mathfrak{p}_{i} N$ and the second equality is by Corollary 3.7. We can refine the filtration $(*)$ by inserting the filtration $\mathcal{F}_{i}$ in it for all $i=1, \ldots, r$. Hence we get the filtration

$$
\mathcal{F}_{M \otimes_{\mathbb{k}} N}: 0=L_{0,0} \subset L_{0,1} \subset \cdots L_{r, s_{r}}=M \otimes_{\mathbb{k}} N
$$

of the $A \otimes_{\mathbb{k}} B$-module $M \otimes_{\mathbb{k}} N$ with cyclic quotients

$$
L_{i, j} / L_{i, j-1} \simeq\left(A \otimes_{\mathbb{k}} B\right) / \mathfrak{P}_{(i, j)},
$$

where $\mathfrak{P}_{(i, j)}:=\mathfrak{p}_{i} \bigotimes_{\mathbb{k}} B+A \otimes_{\mathbb{k}} \mathfrak{q}_{i_{j}}$. Now, let $(i, j) \leq\left(i^{\prime}, j^{\prime}\right)$ where " $\leq$ " is lexicographic order, and $\mathfrak{p}_{i} \otimes_{\mathbb{k}} B+A \otimes_{\mathbb{k}} \mathfrak{q}_{i_{j}} \subseteq \mathfrak{p}_{i^{\prime}} \otimes_{\mathbb{k}} B+A \otimes_{\mathbb{k}} \mathfrak{q}_{i^{\prime} j^{\prime}}$, then by Lemma 3.5, we have $\mathfrak{p}_{i} \subseteq \mathfrak{p}_{i^{\prime}}$ and $\mathfrak{q}_{i_{j}} \subseteq \mathfrak{q}_{i^{\prime}{ }^{\prime}}$. Since $i \leq i^{\prime}$, by pretty cleanness of the $A$-module $M$, we have $\mathfrak{p}_{i}=\mathfrak{p}_{i^{\prime}}$. Also, by cleanness of $B$-module $N$ we conclude that $\mathfrak{q}_{i_{j}}=\mathfrak{q}_{i_{j^{\prime}}^{\prime}}$, as required.

As an application of Corollary 3.8, Corollary 4.8 and also Theorem 4.9, we can see that for a monomial ideal, cleanness and pretty cleanness behave well with respect to reduction modulo a monomial regular element.

Corollary 4.10. Let I be a monomial ideal in the polynomial ring $R=\mathbb{k}\left[x_{1}, \ldots\right.$, $\left.x_{n}\right]$. Let $u \in R$ be a monomial which is regular on $R / I$. Then $R /(I, u)$ is clean (resp. pretty clean) if an only if $R / I$ is clean (resp. pretty clean). 
Proof. Since $I$ is a monomial ideal and $u$ is a monomial regular over $R / I$, we may assume that $R=\mathbb{k}\left[x_{1}, \ldots, x_{r}, x_{r+1}, \ldots, x_{n}\right]$ such that the support of each minimal generator of $I$ is in $\left\{x_{1}, \ldots, x_{r}\right\}$ and the support of the monomial $u$ is in $\left\{x_{r+1}, \ldots, x_{n}\right\}$. Let $S_{1}=\mathbb{k}\left[x_{1}, \ldots, x_{r}\right]$ and $S_{2}=\mathbb{k}\left[x_{r+1}, \ldots, x_{n}\right]$. Then we have

$$
R /(I, u) \simeq S_{1} / I \otimes_{\mathbb{R}} S_{2} /(u)
$$

Since the ideal $(u)$ is a complete intersection monomial ideal, by [9, Proposition $1.2], S_{2} /(u)$ is clean. Therefore, by the isomorphism $R / I \simeq S_{1} / I \otimes_{\mathbb{k}} \mathbb{k}\left[x_{r+1}, \ldots\right.$, $x_{n}$ ], Corollary 3.8, and Corollary 4.8, we get the result.

Remark 4.11. In general, cleanness is not well-behaved with respect to reduction modulo regular elements. To see this, let $A$ be an integral domain which does not satisfy Serre's condition $\left(S_{2}\right)$. For example, let $A$ be an affine simplicial semigroup ring which is not Cohen-Macaulay (cf. [1, Exercise 6.2.8(c)]). Then there exists an element $a \in A$ such that $a A$ has an embedded prime. This implies that $A / a A$ is not clean, but $A$ itself is clean because it is an integral domain.

Acknowledgements. This research was in part supported by a grant from IPM (No. 83130317, No. 86130214, and No. 86130211).

\section{References}

[1] Bruns, W., Herzog, J.: Cohen-Macaulay Rings (revised edition). Cambridge University Press, Cambridge (1998)

[2] Björner, A., Wachs, M., Welker, V.: On Sequentially Cohen-Macaulay complexes and posets. Preprint, arXiv:math.CO/0702788v1

[3] Dress, A.: A new algebraic criterion for shellability. Beiträge Algebra Geom. 34(1), 45-55 (1993)

[4] Duval, A.M.: Algebraic shifting and sequentially Cohen-Macaulay simplicial complexes. Electron. J. Combin. 3 (1996)

[5] Eisenbud, D.: Commutative algebra. With a view toward algebraic geometry. Graduate Texts in Mathematics, vol. 150. Springer, New York (1995)

[6] Fröberg, R.: A note on the Stanley-Reisner ring of a join and of a suspension. Manuscripta Math. 60(1), 89-91 (1988)

[7] Goto, S.: Approximately Cohen-Macaulay rings. J. Algebra 76(1), 214-225 (1982)

[8] Herzog, J., Popescu, D.: Finite filtrations of modules and shellable multicomplexes. Manuscripta Math. 121(3), 385-410 (2006)

[9] Herzog, J., Soleyman Jahan, A., Yassemi, S.: Stanley decompositions and partitionable simplicial complexes. J. Algebraic Combin. 27, 113-125 (2008)

[10] Kang, M.: Almost Cohen-Macaulay modules. Comm. Algebra 29, 781-787 (2001)

[11] Matsumura, H.: Commutative Ring Theory. Translated from the Japanese by M. Reid, 2nd edn. Cambridge University Press, Cambridge (1989)

[12] Miyazaki, M.: Characterizations of Buchsbaum complexes. Manuscripta Math. 63, 245-254 (1989)

[13] Provan, S.J., Billera, L.J.: Decompositions of simplicial complexes related to diameters of convex polyhedra. Math. Oper. Res. 5, 576-594 (1980) 
[14] Schenzel, P.: On the dimension filtration and Cohen-Macaulay filtered modules. Commutative algebra and algebraic geometry (Ferrara), Lecture Notes in Pure and Appl. Math., vol. 206, pp 245-264. Dekker, New York (1999)

[15] Schenzel, P., Trung, N.V., Cuong, N.T.: Verallgemeinerte Cohen-Macaulay-Moduln. (German) Math. Nachr. 85, 57-73 (1978)

[16] Stanley, R.P.: Combinatorics and Commutative Algebra, 2nd edn. Birkhäuser, Basel (1996)

[17] Stückrad, J., Vogel, W.: Buchsbaum Rings and Applications. An Interaction between Algebra, Geometry, and Topology. Mathematical Monographs, Berlin (1986)

[18] Tousi, M., Yassemi, S.: Sequentially Cohen-Macaulay modules under base change. Comm. Algebra 33(11), 3977-3987 (2005)

[19] Vermani, L.R.: An elementary approach to homological algebra. Monographs and Surveys in Pure and Applied Mathematics, vol. 130. Chapman \& Hall/CRC, London (2003)

[20] Wachs, M.L.: Whitney homology of semipure shellable posets. J. Algebraic Combin. 9, 173-207 (1999)

[21] Welker, V.: Constructions preserving evasiveness and collapsibility. Discrete Math. 207(1-3), 243-255 (1999) 